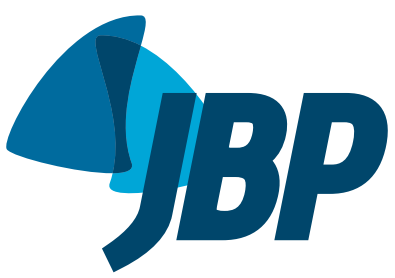

\title{
Eosinophils in COPD: why should I care?
}

\author{
Frederico Leon Arrabal Fernandes ${ }^{1}$
}

What defines a disease? Intuitively, it is very simple to differentiate between disease and health, but it is surprisingly difficult to define what "disease" is. Physicians are extensively trained to recognize signs and symptoms in patients and to attribute such signs and symptoms to a single disease. In practice, this unicist model transforms clinical findings into labels. ${ }^{(1)}$

The problem is that, when patients are given labels, they receive standard treatments that often ignore the particularities of their cases. International consensus guidelines by the Global Initiative for Asthma and the Global Initiative for Chronic Obstructive Lung Disease (GOLD), which cover asthma and COPD, respectively, have been of great value in systematizing care and standardizing the treatment of these conditions, but, in fact, they are simplifications of a multitude of clinical presentations that combine characteristics of both airway diseases. In an attempt to correct this inaccuracy, the concept of an overlap syndrome has been proposed: the asthma and COPD overlap syndrome (ACOS).

Several criteria have been advocated to define patients as having ACOS, and the proportion of such cases can be as high as 30\% among COPD patients. However, this new entity is just another simplification of a complex set of clinical characteristics, and its relevance has been questioned. (2)

It has long been debated whether asthma and COPD are distinct diseases or two sides of the same coin. The presence of individuals in whom a diagnosis of either asthma or COPD cannot be established, as well as of individuals who present with characteristics of both diseases, reinforces the concept that asthma and COPD are spectra of the same disease. Not even biological markers, such as sputum examination, can definitively separate these two conditions.

$A$ recent study compared sputum characteristics in COPD and in asthma. It was possible to divide the results into three groups: one group with clear Th2 eosinophilic-predominant inflammation; one group with a neutrophil-predominant profile; and one group, comprising one third of the patients evaluated, in which there was no clear distinction between the two diagnoses. ${ }^{(3)}$

Another study evaluated the presence of sputum eosinophilia in COPD patients, who were then treated with a systemic corticosteroid for two weeks. ${ }^{(4)}$ Eosinophilia was a marker of clinical response to the corticosteroid. It has been demonstrated that a systematic evaluation of sputum eosinophilia can help to prevent exacerbations. ${ }^{(5)}$

It has been established that there is a strong correlation between sputum eosinophilia and peripheral eosinophilia in COPD. ${ }^{(6)}$ Peripheral eosinophilia is a biomarker of response to inhaled corticosteroids (ICs). Subgroup analyses performed in three large-scale studies showed that the benefit of ICs in preventing exacerbations was found only in the subgroup of patients with an eosinophil count greater than $2 \% .{ }^{(7)}$ The response to ICs in terms of the rate of decline in pulmonary function also seems to be marked by eosinophilia. When treated with ICs, patients with an eosinophil count greater than $2 \%$ showed a marked reduction in the annual rate of decline in $\mathrm{FEV}_{1}$ (from $74.5 \mathrm{~mL}$ to $40.6 \mathrm{~mL}$ ). In the absence of this marker, there was no change in the rate of decline in pulmonary function. ${ }^{(7)}$

In their study published in this issue of the JBP, Queiroz et al. ${ }^{(8)}$ investigated the inflammatory profile in the sputum from 37 patients with COPD, dividing them into those with and those without a bronchodilator response. The principal findings were as follows: patients with a bronchodilator response had greater sputum eosinophilia, regardless of an asthma diagnosis or clinical and laboratory markers of atopy (including the levels of measured cytokines); and the proportion of eosinophils in the sputum correlated inversely with $\mathrm{FEV}_{1}$, especially in patients with GOLD stage III COPD. Paradoxically, there were no eosinophils in the sputum from patients with GOLD stage IV COPD.

Despite the limitations imposed by the cross-sectional design of and the small number of recruited patients to the study by Queiroz et al.,(8) its results lead to important considerations. The first of these considerations is that the evaluation of eosinophilia in COPD does not depend on an asthma diagnosis. The presence of eosinophils in the sputum, rather than being only another finding in patients with a history or characteristics of atopy, is a strong marker of severity and bronchodilator response. This underscores the need for laboratory characterization of the type of inflammatory process.

Another interesting finding is that, in agreement with previous studies, the presence of eosinophils in the airway is associated with disease severity and progression, but, at some point in the natural history of COPD, the inflammatory process is reduced, with its markers becoming less evident as $\mathrm{FEV}_{1}$ decreases. This finding helps to demonstrate the complexity of COPD, which behaves differently and apparently in a contradictory manner, in the various stages of the disease. Because of that, clinical trials have shown the benefit of treatment of earlier disease (stages II or III) when the inflammatory process is intense. ${ }^{(9)}$

Rather than labeling diseases, ignoring that variations of a rule are more common than the rule itself, we should understand that there are objective markers that help to 
construct the clinical and laboratory profile, allowing the proper choice of treatment. Eosinophilia has been establishing itself as one of the most important markers.

The approach to treating obstructive airway diseases based on labels disregards the biological complexity of these conditions and ignores the multiplicity of clinical presentations. It leads to underprescription or overprescription of medications and limits the progress of research, given that patients who do not fit any one definition are excluded from clinical trials. By taking into account eosinophils in COPD, we are ensuring treatment with a higher likelihood of response and fewer adverse effects, thereby offering personalized quality medicine. ${ }^{(10)}$

\section{REFERENCES}

1. Scully JL. What is a disease? EMBO reports. 2004;5(7):650-3. http:// dx.doi.org/10.1038/sj.embor.7400195

2. Bujarski S, Parulekar AD, Sharafkhaneh A, Hanania NA. The asthma COPD overlap syndrome (ACOS). Curr Allergy Asthma Rep. 2015;15(3):509. http://dx.doi.org/10.1007/s11882-014-0509-6

3. Ghebre MA, Bafadhel M, Desai D, Cohen SE, Newbold P, Rapley $L$, et al. Biological clustering supports both "Dutch" and "British" hypotheses of asthma and chronic obstructive pulmonary disease. J Allergy Clin Immunol. 2015;135(1):63-72. http://dx.doi.org/10.1016/j. jaci.2014.06.035

4. Brightling CE, Monteiro W, Ward R, Parker D, Morgan MD Wardlaw AJ, et al. Sputum eosinophilia and short-term response to prednisolone in chronic obstructive pulmonary disease: a randomised controlled trial. Lancet. 2000;356(9240):1480-5. http:// dx.doi.org/10.1016/S0140-6736(00)02872-5

5. Siva R, Green RH, Brightling CE, Shelley M, Hargadon B, McKenna S, et al. Eosinophilic airway inflammation and exacerbations of COPD: a randomised controlled trial. Eur Resp J. 2007;29(5):906-13. http:// dx.doi.org/10.1183/09031936.00146306

6. Rufino R, Costa CH, Souza HS, Madi K, Silva JR. Induced sputum and

peripheral blood cell profile in chronic obstructive pulmonary disease. J Bras Pneumol. 2007;33(5):510-8

7. Pascoe S, Locantore N, Dransfield MT, Barnes NC, Pavord ID. Blood eosinophil counts, exacerbations, and response to the addition of inhaled fluticasone furoate to vilanterol in patients with chronic obstructive pulmonary disease: a secondary analysis of data from two parallel randomised controlled trials. Lancet Respir Med. 2015;3(6):435-42. http://dx.doi.org/10.1016/S2213-2600(15)00106-X

8. Queiroz CF, Lemos AC, Bastos ML, Neves MC, Camelier AA Carvalho NB, et al. Inflammatory and immunological profiles in patients with COPD: relationship with FEV1 reversibility. J Bras Pneumol. 2016;42(4):241-7.

9. Eagan TM, Ueland T, Wagner PD, Hardie JA, Mollnes TE, Damas JK, et al. Systemic inflammatory markers in COPD: results from the Bergen COPD Cohort Study. Eur Resp J. 2010;35(3):540-8. http:// dx.doi.org/10.1183/09031936.00088209

10. Agusti A, Anto JM, Auffray C, Barbe F, Barreiro E, Dorca J, et al Personalized respiratory medicine: exploring the horizon, addressing the issues. Summary of a BRN-AJRCCM workshop held in Barcelona on June 12, 2014. Am J Respir Crit Care Med. 2015;191(4):391-401. http://dx.doi.org/10.1164/rccm.201410-1935PP 